\title{
Some Socio-Economic Aspects of Consumers in Unorganized Markets of Kolkata City, India
}

\author{
S. Sinha* and Md. H. Ali \\ Department of Agricultural Economics, Bidhan Chandra Krishi Viswavidyalaya \\ Mohanpur, Distt. Nadia, West Bengal, India \\ *Corresponding author
}

Keywords

Customers,

Unorganized,

Retail, Dry

Commodities,

Wet commodities

Article Info

Accepted:

12 March 2020

Available Online:

10 April 2020

\section{A B S T R A C T}

Consumers' perception is defined as the study of how an individual customer, groups of customer select, buy, use, and dispose ideas, goods, and services that cater their needs and wants. It refers to the actions of the consumers in the marketplace and the underlying motives for those actions. Rathod et al., (2008), focused on finding the difference of criteria for selection of retail store among customers. Primary data was collected from seven big cities of Gujarat by quota sampling. The findings showed that the choice criteria for selecting a particular retail outlet, in case of multi brand retail outlets differed. The availability of variety was given the highest importance by customers, second priority was given to service quality and third most important criteria was availability of variety and fourth was shopping convenience. The study intends to examine the consumers' perception for marketing differential agro-commodities in the unorganized markets. The study revealed that the maximum percentage of customers purchases wet commodities (vegetables and fruits) from traditional markets whereas in case of dry (grocery) commodities they prefer organized outlets.

\section{Introduction}

Retailing is the final stage of any economic activity. It includes every sale to final consumers ranging from cars to apparel to meals at restaurants to movie tickets.

\section{Unorganized retailing (supply-based)}

Unorganized retailing is also known as the traditional formats of low-cost retailing, for example, the local kirana shops, owner managed general stores, convenience stores, hand cart and pavement vendors, etc. These types of market are characterized by typically small retailers who tend to avoid taxes and do not follow labor law. These markets are often found in developing countries.

Yoo et al., (2006) identified the attributes that consumers consider to be most important when making format choices, and also the 
demographic characteristics of these consumers in grocery retailers that operate within the US. The knowledge generated with the study would help in decisions related to retail formats.

The exploration of the theoretical background found store image attributes suggested by many previous researchers as important determinants of store loyalty and also to identify which store image attributes affect store loyalty significantly according to retail type respectively.

The Indian retail industry has emerged as one of the most dynamic and fast-growing industries owing to the emergence of several new stakeholders. India is the world's fifthlargest global destination in the retail space.

Consumer familiarity that runs from generation to generation is one big advantage for the traditional traders. Consumer familiarity that runs from generation to generation is one big advantage for the traditional traders. (Source: https://www.ibef.org/industry/retailindia.aspx)

In view of the above, it is pertinent to study the consumer behavior of Kolkata with regard to agricultural commodities which is defined as the study of how an individual customer, groups or organizations select, buy, use, and dispose ideas, goods, and services to satisfy their needs and wants. It refers to the actions of the consumers in the marketplace and the underlying motives for those actions.

To examine consumers' perception for marketing differential agro-commodities in the unorganized markets.

\section{Materials and Methods}

The study is strictly based on primary information collected through canvassing pre- ordained questionnaire among the selected traditional market customers to fulfill the objectives set out. Relevant data are collected from primary sources. The area of survey operation should be carried out within 30 kilometer radius from central Kolkata. The study area is divided into 5 parts (Clusters):

Sealdah

Gariahat

South Dumdum

Gopalpur

Newtown

\section{Selection of study area}

Within the different parts of the study area, we have purposively selected 5 clusters where all the selected clusters consist of traditional retail outlets dealing with both agricultural and non-agricultural commodities.

There are altogether 47 traditional markets distributed over the 5 selected clusters. Out of 47 traditional markets we have selected proportionately 12 markets $(25 \%$ the number of traditional markets) for the collection of information. Thus our selected traditional markets are as follows:

Sealdah - Entally market, Baithakhana market, Jhanbajar market

Gariahat -Gariahat market, Panditya market

South Dumdum- Nagerbajar Market, Subhashnagar Market, Kalindi Market

Gopalpur- Teghoria Market, Baguihati Market

Newtown- Pakpole Market, Newtown Market

\section{Selection of sample}

Our unit of observation is customers. The selection of customers is made by approaching the customers available in our particular selected shop to provide us the necessary information. This kind of selection may be termed as convenience sampling but 
here some random nature is attached here with. From each of the markets we have randomly selected from 20 customers and collected information from them in each of the traditional markets. Thus we have 240 $(20 * 12=240)$ customers.

\section{Collection of data}

Collection of data shall be made by visiting and interrogating each and every sample respondents with the help of a set of pretested survey schedules. Both tabular and statistical analysis shall be made to analyze and interpret the data in order to get desirable results from the study.

\section{Results and Discussion}

Results of the research study are presented in the form of tabular analysis which is as follows:

Table 1 shows the age-wise and sex-wise frequency distribution of the traditional market customers in all the traditional markets across all the clusters. It is understood from the table that the customer's age group is classified into 4 categories, such as, below 20 years age group, 21 to 40 years, 41 to 60 years and above 60 years age group, out of which, the maximum percentage of the male customers belong to 21 to 40 whereas maximum percentage of female customers belong to 20-40 years age groups.

The Table 2 shows the frequency distribution of traditional market customers on the basis of family size in all the traditional markets across all the clusters. From the table it appears that the family size is mainly grouped into 3 categories i.e up to 3 members, 4 to 6 members and 7 to 9 and more.

It is observed from the study that all family sizes are found but the maximum percentage of traditional market customers have family size of 4-6 members followed by up to 3 members and family size of 7 to 9 members.

The Table 3 reveals the frequency distribution of traditional market customers on the basis on the basis of educational qualification in all the traditional markets across all the clusters. It is clearly understood from the table that the percentage of graduates' customers in traditional market is higher compared to percentage of customers who are have passed higher secondary examination and below higher secondary level.

The Table 4 demonstrates the frequency distribution of traditional market consumers on the basis of occupation in all the traditional markets across all the clusters. From the table it appears that the maximum percentages of customers are private sectors service holders followed by in government service holders.

The Table 5 demonstrates the frequency distribution of traditional market consumers on the basis of family income in all the traditional markets across all the clusters.

It is observed from the table that the maximum percentage of family income belongs to family income range from rupees 25001 to 50000 followed by lower percentage of income group rupees 15001 to 25000 .

The Table 6 reveals the frequency distribution of customers in traditional markets on the basis of distance between the residence and the respective closest traditional market in all the clusters of Kolkata. It can be observed from the table that the maximum percentage of customer from both the markets resides between $2-2.9 \mathrm{~km}$ followed by $1-1.9 \mathrm{~km}$.

The Table 7 demonstrates the frequency distribution of the traditional market customers on the basis type of commodities bought by them in all the traditional markets 
across all the clusters in Kolkata. It is evident from the table that the maximum percentage of consumers purchases wet commodities (vegetables and fruits) compared to percentage of customers who purchases dry commodities (grocery) and purchase both type of commodities (dry and wet) from the traditional markets.

Table.1 Age wise and sex wise distribution of the traditional customers (Cluster-Pooled)

\begin{tabular}{|c|c|c|c|c|c|c|c|c|c|c|c|}
\hline & \multicolumn{2}{|c|}{ Below 20} & \multicolumn{2}{|c|}{ 20-40years } & \multicolumn{2}{|c|}{ 41-60years } & \multicolumn{2}{|c|}{ More than 60} & \multicolumn{2}{|c|}{ Sub Total } & \multirow[t]{2}{*}{ Total } \\
\hline & $\mathbf{M}$ & $\mathbf{F}$ & $\mathbf{M}$ & F & $\mathbf{M}$ & F & $\mathbf{M}$ & F & $\mathbf{M}$ & F & \\
\hline $\begin{array}{l}\text { Cluster - } \\
\text { Sealdah }\end{array}$ & $\begin{array}{c}0 \\
(0.00)\end{array}$ & $\begin{array}{c}0 \\
(0.00)\end{array}$ & $\begin{array}{c}11 \\
(18.33)\end{array}$ & $\begin{array}{c}19 \\
(31.66)\end{array}$ & $\begin{array}{c}18 \\
(30)\end{array}$ & $\begin{array}{c}11 \\
(18.33)\end{array}$ & $\begin{array}{c}3 \\
(5)\end{array}$ & $\begin{array}{c}0 \\
(0.00)\end{array}$ & $\begin{array}{c}32 \\
(53.33)\end{array}$ & $\begin{array}{c}28 \\
(46.66)\end{array}$ & $\begin{array}{c}60 \\
(100)\end{array}$ \\
\hline $\begin{array}{l}\text { Cluster- } \\
\text { Gariahat }\end{array}$ & $\begin{array}{c}0 \\
(0.00)\end{array}$ & $\begin{array}{c}0 \\
(0.00)\end{array}$ & $\begin{array}{c}8 \\
(20)\end{array}$ & $\begin{array}{c}12 \\
(30)\end{array}$ & $\begin{array}{c}14 \\
(35)\end{array}$ & $\begin{array}{c}6 \\
(15)\end{array}$ & $\begin{array}{c}4 \\
(10)\end{array}$ & $\begin{array}{c}0 \\
(0.00)\end{array}$ & $\begin{array}{c}26 \\
(65)\end{array}$ & $\begin{array}{c}14 \\
(35)\end{array}$ & $\begin{array}{c}40 \\
(100)\end{array}$ \\
\hline $\begin{array}{l}\text { Cluster- } \\
\text { South } \\
\text { Dumdum }\end{array}$ & $\begin{array}{c}0 \\
(0.00)\end{array}$ & $\begin{array}{c}0 \\
(0.00)\end{array}$ & $\begin{array}{c}16 \\
(26.66)\end{array}$ & $\begin{array}{c}10 \\
(16.66)\end{array}$ & $\begin{array}{c}16 \\
(26.66)\end{array}$ & $\begin{array}{c}8 \\
(13.33)\end{array}$ & $\begin{array}{c}4 \\
(6.66)\end{array}$ & $\begin{array}{c}0 \\
(0.00)\end{array}$ & $\begin{array}{c}36 \\
(60)\end{array}$ & $\begin{array}{c}24 \\
(40)\end{array}$ & $\begin{array}{c}60 \\
(100)\end{array}$ \\
\hline $\begin{array}{l}\text { Cluster- } \\
\text { Gopalpur }\end{array}$ & $\begin{array}{c}0 \\
(0.00)\end{array}$ & $\begin{array}{c}0 \\
(0.00)\end{array}$ & $\begin{array}{c}10 \\
(25)\end{array}$ & $\begin{array}{c}11 \\
(27.5)\end{array}$ & $\begin{array}{c}14 \\
(35)\end{array}$ & $\begin{array}{c}6 \\
(15)\end{array}$ & $\begin{array}{c}3 \\
(7.5)\end{array}$ & $\begin{array}{c}0 \\
(0.00)\end{array}$ & $\begin{array}{c}27 \\
(67.5)\end{array}$ & $\begin{array}{c}13 \\
(32.5)\end{array}$ & $\begin{array}{c}40 \\
(100)\end{array}$ \\
\hline $\begin{array}{l}\text { Cluster- } \\
\text { Newtown }\end{array}$ & $\begin{array}{c}0 \\
(0.00)\end{array}$ & $\begin{array}{c}0 \\
(0.00) \\
\end{array}$ & $\begin{array}{c}7 \\
(17.5) \\
\end{array}$ & $\begin{array}{c}15 \\
(37.5)\end{array}$ & $\begin{array}{c}14 \\
(35)\end{array}$ & $\begin{array}{c}5 \\
(12.5) \\
\end{array}$ & $\begin{array}{c}0 \\
(0.00)\end{array}$ & $\begin{array}{c}0 \\
(0.00) \\
\end{array}$ & $\begin{array}{c}21 \\
(52.5)\end{array}$ & $\begin{array}{c}19 \\
(47.5)\end{array}$ & $\begin{array}{c}40 \\
(100)\end{array}$ \\
\hline $\begin{array}{c}\text { Grand } \\
\text { Total }\end{array}$ & $\begin{array}{c}0 \\
(0.00)\end{array}$ & $\begin{array}{c}0 \\
(0.00)\end{array}$ & $\begin{array}{c}52 \\
(21.66)\end{array}$ & $\begin{array}{c}67 \\
(27.91)\end{array}$ & $\begin{array}{c}76 \\
(31.66)\end{array}$ & $\begin{array}{c}36 \\
(15)\end{array}$ & $\begin{array}{c}14 \\
(5.83)\end{array}$ & $\begin{array}{c}0 \\
(0.00)\end{array}$ & $\begin{array}{c}142 \\
(59.16)\end{array}$ & $\begin{array}{c}98 \\
(40.83)\end{array}$ & $\begin{array}{c}240 \\
(100)\end{array}$ \\
\hline
\end{tabular}

Figures in the parentheses indicate the percentages to the total (row wise)

Table.2 Frequency distribution of traditional customers on the basis of family size (Cluster-Pooled)

\begin{tabular}{|c|c|c|c|c|c|c|c|c|c|}
\hline & \multicolumn{2}{|c|}{$\begin{array}{c}\text { Up to } 3 \\
\text { Members }\end{array}$} & \multicolumn{2}{|c|}{ 4-6 Members } & \multicolumn{2}{|c|}{ 7-9 Members } & \multicolumn{2}{|c|}{ Sub Total } & \multirow[t]{2}{*}{ Total } \\
\hline & M & $\mathbf{F}$ & $\mathbf{M}$ & $\mathbf{F}$ & M & $\mathbf{F}$ & M & $\mathbf{F}$ & \\
\hline $\begin{array}{l}\text { Cluster - } \\
\text { Sealdah }\end{array}$ & $\begin{array}{c}11 \\
(11.33)\end{array}$ & $\begin{array}{c}6 \\
(10)\end{array}$ & $\begin{array}{c}13 \\
(21.66)\end{array}$ & $\begin{array}{c}21 \\
(35)\end{array}$ & $\begin{array}{c}5 \\
(8.33)\end{array}$ & $\begin{array}{c}4 \\
(6.66)\end{array}$ & $\begin{array}{c}32 \\
(53.33)\end{array}$ & $\begin{array}{c}28 \\
(46.66)\end{array}$ & $\begin{array}{c}60 \\
(100)\end{array}$ \\
\hline $\begin{array}{l}\text { Cluster- } \\
\text { Gariahat }\end{array}$ & $\begin{array}{c}11 \\
(27.5)\end{array}$ & $\begin{array}{c}3 \\
(7.5)\end{array}$ & $\begin{array}{c}12 \\
(30)\end{array}$ & $\begin{array}{c}11 \\
(27.5)\end{array}$ & $\begin{array}{c}3 \\
(7.5)\end{array}$ & $\begin{array}{c}0 \\
(0.00)\end{array}$ & $\begin{array}{c}26 \\
(65)\end{array}$ & $\begin{array}{c}14 \\
(35)\end{array}$ & $\begin{array}{c}40 \\
(100)\end{array}$ \\
\hline $\begin{array}{l}\text { Cluster- } \\
\text { South } \\
\text { Dumdum }\end{array}$ & $\begin{array}{c}16 \\
(26.66)\end{array}$ & $\begin{array}{c}15 \\
(25)\end{array}$ & $\begin{array}{c}18 \\
(30)\end{array}$ & $\begin{array}{c}9 \\
(15)\end{array}$ & $\begin{array}{c}2 \\
(3.33)\end{array}$ & $\begin{array}{c}0 \\
(0.00)\end{array}$ & $\begin{array}{c}36 \\
(60)\end{array}$ & $\begin{array}{c}24 \\
(40)\end{array}$ & $\begin{array}{c}60 \\
(100)\end{array}$ \\
\hline $\begin{array}{l}\text { Cluster- } \\
\text { Gopalpur }\end{array}$ & $\begin{array}{c}11 \\
(27.5)\end{array}$ & $\begin{array}{c}4 \\
(10)\end{array}$ & $\begin{array}{c}13 \\
(32.5)\end{array}$ & $\begin{array}{c}9 \\
(22.5)\end{array}$ & $\begin{array}{c}3 \\
(7.5)\end{array}$ & $\begin{array}{c}0 \\
(0.00)\end{array}$ & $\begin{array}{c}27 \\
(67.5)\end{array}$ & $\begin{array}{c}13 \\
(32.5)\end{array}$ & $\begin{array}{c}40 \\
(100)\end{array}$ \\
\hline $\begin{array}{l}\text { Cluster- } \\
\text { Newtown }\end{array}$ & $\begin{array}{c}10 \\
(25)\end{array}$ & $\begin{array}{c}3 \\
(7.5)\end{array}$ & $\begin{array}{c}8 \\
(20)\end{array}$ & $\begin{array}{c}12 \\
(30)\end{array}$ & $\begin{array}{c}3 \\
(7.5)\end{array}$ & $\begin{array}{c}4 \\
(10)\end{array}$ & $\begin{array}{c}21 \\
(52.5)\end{array}$ & $\begin{array}{c}19 \\
(47.5)\end{array}$ & $\begin{array}{c}40 \\
(100)\end{array}$ \\
\hline $\begin{array}{c}\text { Grand } \\
\text { Total }\end{array}$ & $\begin{array}{c}59 \\
(24.58)\end{array}$ & $\begin{array}{c}31 \\
(12.91)\end{array}$ & $\begin{array}{c}64 \\
(26.66)\end{array}$ & $\begin{array}{c}62 \\
(25.83)\end{array}$ & $\begin{array}{c}15 \\
(6.25)\end{array}$ & $\begin{array}{c}8 \\
(3.33)\end{array}$ & $\begin{array}{c}142 \\
(59.16)\end{array}$ & $\begin{array}{c}98 \\
(40.83)\end{array}$ & $\begin{array}{c}240 \\
(100)\end{array}$ \\
\hline
\end{tabular}

Figures in the parentheses indicate the percentages to the total (row wise) 
Table.3 Frequency distribution of traditional customers on the basis of educational qualification (Cluster Pooled)

\begin{tabular}{|c|c|c|c|c|c|c|c|c|c|c|c|}
\hline & \multicolumn{2}{|c|}{ Graduate } & \multicolumn{2}{|c|}{$\begin{array}{c}\text { Higher } \\
\text { Secondary }\end{array}$} & \multicolumn{2}{|c|}{$\begin{array}{c}\text { Below } \\
\text { Higher } \\
\text { Secondary }\end{array}$} & \multicolumn{2}{|c|}{ Illiterate } & \multicolumn{2}{|c|}{ Sub Total } & \multirow[t]{2}{*}{ Total } \\
\hline & $\mathbf{M}$ & $\mathbf{F}$ & $\mathbf{M}$ & $\mathbf{F}$ & $\mathbf{M}$ & $\mathbf{F}$ & $\mathbf{M}$ & $\mathbf{F}$ & $\mathbf{M}$ & $\mathbf{F}$ & \\
\hline $\begin{array}{l}\text { Cluster - } \\
\text { Sealdah }\end{array}$ & $\begin{array}{c}13 \\
(21.66)\end{array}$ & $\begin{array}{c}10 \\
(16.66)\end{array}$ & $\begin{array}{c}12 \\
(20)\end{array}$ & $\begin{array}{c}10 \\
(16.66)\end{array}$ & $\begin{array}{c}6 \\
(10)\end{array}$ & $\begin{array}{c}6 \\
(10)\end{array}$ & $\begin{array}{c}1 \\
(1.66)\end{array}$ & $\begin{array}{c}2 \\
(3.33)\end{array}$ & $\begin{array}{c}32 \\
(53.33)\end{array}$ & $\begin{array}{c}28 \\
(46.66)\end{array}$ & $\begin{array}{c}60 \\
(100)\end{array}$ \\
\hline $\begin{array}{l}\text { Cluster- } \\
\text { Gariahat }\end{array}$ & $\begin{array}{c}20 \\
(50)\end{array}$ & $\begin{array}{c}14 \\
(35)\end{array}$ & $\begin{array}{c}6 \\
(15)\end{array}$ & $\begin{array}{c}2 \\
(5)\end{array}$ & $\begin{array}{c}0 \\
(0.00)\end{array}$ & $\begin{array}{c}0 \\
(0.00)\end{array}$ & $\begin{array}{c}0 \\
(0.00)\end{array}$ & $\begin{array}{c}0 \\
(0.00)\end{array}$ & $\begin{array}{c}26 \\
(65)\end{array}$ & $\begin{array}{c}14 \\
(35)\end{array}$ & $\begin{array}{c}40 \\
(100)\end{array}$ \\
\hline $\begin{array}{l}\text { Cluster- } \\
\text { South } \\
\text { Dumdum }\end{array}$ & $\begin{array}{c}28 \\
(46.66)\end{array}$ & $\begin{array}{c}16 \\
(26.66)\end{array}$ & $\begin{array}{c}8 \\
(13.33)\end{array}$ & $\begin{array}{c}5 \\
(8.33)\end{array}$ & $\begin{array}{c}0 \\
(0.00)\end{array}$ & $\begin{array}{c}0 \\
(0.00)\end{array}$ & $\begin{array}{c}0 \\
(0.00)\end{array}$ & $\begin{array}{c}0 \\
(0.00)\end{array}$ & $\begin{array}{c}36 \\
(60)\end{array}$ & $\begin{array}{c}24 \\
(40)\end{array}$ & $\begin{array}{c}60 \\
(100)\end{array}$ \\
\hline $\begin{array}{l}\text { Cluster- } \\
\text { Gopalpur }\end{array}$ & $\begin{array}{c}20 \\
(50)\end{array}$ & $\begin{array}{c}11 \\
(27.5)\end{array}$ & $\begin{array}{c}7 \\
(17.5)\end{array}$ & $\begin{array}{c}2 \\
(5)\end{array}$ & $\begin{array}{c}0 \\
(0.00)\end{array}$ & $\begin{array}{c}0 \\
(0.00)\end{array}$ & $\begin{array}{c}0 \\
(0.00)\end{array}$ & $\begin{array}{c}0 \\
(0.00)\end{array}$ & $\begin{array}{c}27 \\
(67.5)\end{array}$ & $\begin{array}{c}13 \\
(32.5)\end{array}$ & $\begin{array}{c}40 \\
(100)\end{array}$ \\
\hline $\begin{array}{l}\text { Cluster- } \\
\text { Newtown }\end{array}$ & $\begin{array}{c}5 \\
(12.5)\end{array}$ & $\begin{array}{c}15 \\
(37.5)\end{array}$ & $\begin{array}{c}9 \\
(22.5)\end{array}$ & $\begin{array}{c}3 \\
(7.5)\end{array}$ & $\begin{array}{c}5 \\
(12.5)\end{array}$ & $\begin{array}{c}1 \\
(2.5)\end{array}$ & $\begin{array}{c}2 \\
(5)\end{array}$ & $\begin{array}{c}0 \\
(0.00)\end{array}$ & $\begin{array}{c}21 \\
(52.5)\end{array}$ & $\begin{array}{c}19 \\
(47.5)\end{array}$ & $\begin{array}{c}40 \\
(100)\end{array}$ \\
\hline $\begin{array}{c}\text { Grand } \\
\text { Total }\end{array}$ & $\begin{array}{c}86 \\
(35)\end{array}$ & $\begin{array}{c}66 \\
(27.5) \\
\end{array}$ & $\begin{array}{c}42 \\
(17.5)\end{array}$ & $\begin{array}{c}22 \\
(9.16)\end{array}$ & $\begin{array}{c}11 \\
(4.58)\end{array}$ & $\begin{array}{c}7 \\
(2.91) \\
\end{array}$ & $\begin{array}{c}3 \\
(1.25)\end{array}$ & $\begin{array}{c}2 \\
(0.83) \\
\end{array}$ & $\begin{array}{c}142 \\
(59.16)\end{array}$ & $\begin{array}{c}98 \\
(40.83)\end{array}$ & $\begin{array}{c}240 \\
(100)\end{array}$ \\
\hline
\end{tabular}

Figures in the parentheses indicate the percentages to the total (row wise)

Table.4 Frequency distribution of traditional market consumers on the basis of occupation (Cluster Pooled)

\begin{tabular}{|c|c|c|c|c|c|c|c|c|c|c|c|c|c|c|c|}
\hline & \multicolumn{2}{|c|}{ Govt service } & \multicolumn{2}{|c|}{ Private service } & \multicolumn{2}{|c|}{ Businessman } & \multicolumn{2}{|c|}{ Labourer } & \multicolumn{2}{|c|}{ Housewife } & \multicolumn{2}{|c|}{ Dependent } & \multicolumn{2}{|c|}{ Sub Total } & \multirow[t]{2}{*}{ Total } \\
\hline & $\mathbf{M}$ & F & M & $\mathbf{F}$ & M & $\mathbf{F}$ & M & F & M & F & M & $\mathbf{F}$ & $\mathbf{M}$ & $\mathbf{F}$ & \\
\hline $\begin{array}{l}\text { Cluster - } \\
\text { Sealdah }\end{array}$ & $\begin{array}{c}11 \\
(18.33)\end{array}$ & $\begin{array}{c}6 \\
(10)\end{array}$ & $\begin{array}{c}14 \\
(23.33)\end{array}$ & $\begin{array}{c}7 \\
(11.66)\end{array}$ & $\begin{array}{c}4 \\
(6.66)\end{array}$ & $\begin{array}{c}0 \\
(0.00)\end{array}$ & $\begin{array}{c}2 \\
(3.33)\end{array}$ & $\begin{array}{c}1 \\
(1.66)\end{array}$ & $\begin{array}{c}0 \\
(0.00)\end{array}$ & $\begin{array}{c}14 \\
(23.33)\end{array}$ & $\begin{array}{c}0 \\
(0.00)\end{array}$ & $\begin{array}{c}0 \\
(0.00)\end{array}$ & $\begin{array}{c}32 \\
(53.33)\end{array}$ & $\begin{array}{c}28 \\
(46.66)\end{array}$ & $\begin{array}{c}60 \\
(100)\end{array}$ \\
\hline $\begin{array}{l}\text { Cluster- } \\
\text { Gariahat }\end{array}$ & $\begin{array}{c}6 \\
(15)\end{array}$ & $\begin{array}{c}6 \\
(15)\end{array}$ & $\begin{array}{c}11 \\
(27.5)\end{array}$ & $\begin{array}{c}2 \\
(5)\end{array}$ & $\begin{array}{c}9 \\
(22.5) \\
\end{array}$ & $\begin{array}{c}0 \\
(0.00)\end{array}$ & $\begin{array}{c}0 \\
(0.00) \\
\end{array}$ & $\begin{array}{c}0 \\
(0.00)\end{array}$ & $\begin{array}{c}0 \\
(0.00)\end{array}$ & $\begin{array}{c}6 \\
(15)\end{array}$ & $\begin{array}{c}0 \\
(0.00)\end{array}$ & $\begin{array}{c}0 \\
(0.00)\end{array}$ & $\begin{array}{c}26 \\
(65)\end{array}$ & $\begin{array}{c}14 \\
(35)\end{array}$ & $\begin{array}{c}40 \\
(100)\end{array}$ \\
\hline $\begin{array}{l}\text { Cluster- } \\
\text { South } \\
\text { Dumdum }\end{array}$ & $\begin{array}{c}15 \\
(25)\end{array}$ & $\begin{array}{c}2 \\
(3.33)\end{array}$ & $\begin{array}{c}13 \\
(21.66)\end{array}$ & $\begin{array}{c}4 \\
(6.66)\end{array}$ & $\begin{array}{c}8 \\
(13.33)\end{array}$ & $\begin{array}{c}0 \\
(0.00)\end{array}$ & $\begin{array}{c}0 \\
(0.00)\end{array}$ & $\begin{array}{c}0 \\
(0.00)\end{array}$ & $\begin{array}{c}0 \\
(0.00)\end{array}$ & $\begin{array}{c}18 \\
(30)\end{array}$ & $\begin{array}{c}0 \\
(0.00)\end{array}$ & $\begin{array}{c}0 \\
(0.00)\end{array}$ & $\begin{array}{c}36 \\
(60)\end{array}$ & $\begin{array}{c}24 \\
(40)\end{array}$ & $\begin{array}{c}60 \\
(100)\end{array}$ \\
\hline $\begin{array}{l}\text { Cluster- } \\
\text { Gopalpur }\end{array}$ & $\begin{array}{c}6 \\
(15)\end{array}$ & $\begin{array}{c}5 \\
(12.5)\end{array}$ & $\begin{array}{c}12 \\
(30)\end{array}$ & $\begin{array}{c}2 \\
(5)\end{array}$ & $\begin{array}{c}9 \\
(22.5)\end{array}$ & $\begin{array}{c}0 \\
(0.00)\end{array}$ & $\begin{array}{c}0 \\
(0.00)\end{array}$ & $\begin{array}{c}0 \\
(0.00)\end{array}$ & $\begin{array}{c}0 \\
(0.00)\end{array}$ & $\begin{array}{c}6 \\
(15)\end{array}$ & $\begin{array}{c}0 \\
(0.00)\end{array}$ & $\begin{array}{c}0 \\
(0.00)\end{array}$ & $\begin{array}{c}27 \\
(67.5)\end{array}$ & $\begin{array}{c}13 \\
(32.5)\end{array}$ & $\begin{array}{c}40 \\
(100)\end{array}$ \\
\hline $\begin{array}{l}\text { Cluster- } \\
\text { Newtown }\end{array}$ & $\begin{array}{c}6 \\
(15)\end{array}$ & $\begin{array}{c}6 \\
(15)\end{array}$ & $\begin{array}{c}11 \\
(27.5)\end{array}$ & $\begin{array}{c}6 \\
(15)\end{array}$ & $\begin{array}{c}2 \\
(5)\end{array}$ & $\begin{array}{c}1 \\
(2.5)\end{array}$ & $\begin{array}{c}2 \\
(5)\end{array}$ & $\begin{array}{c}0 \\
(0.00)\end{array}$ & $\begin{array}{c}0 \\
(0.00)\end{array}$ & $\begin{array}{c}6 \\
(15)\end{array}$ & $\begin{array}{c}0 \\
(0.00)\end{array}$ & $\begin{array}{c}0 \\
(0.00)\end{array}$ & $\begin{array}{c}21 \\
(52.5)\end{array}$ & $\begin{array}{c}19 \\
(47.5)\end{array}$ & $\begin{array}{c}40 \\
(100)\end{array}$ \\
\hline $\begin{array}{c}\text { Grand } \\
\text { Total }\end{array}$ & $\begin{array}{c}44 \\
(18.33)\end{array}$ & $\begin{array}{c}25 \\
(10.41)\end{array}$ & $\begin{array}{c}61 \\
(25.41)\end{array}$ & $\begin{array}{c}21 \\
(8.75)\end{array}$ & $\begin{array}{c}32 \\
(13.33)\end{array}$ & $\begin{array}{c}1 \\
(0.41)\end{array}$ & $\begin{array}{c}4 \\
(1.66)\end{array}$ & $\begin{array}{c}1 \\
(0.41)\end{array}$ & $\begin{array}{c}0 \\
(0.00)\end{array}$ & $\begin{array}{c}50 \\
(20.83)\end{array}$ & $\begin{array}{c}0 \\
(0.00)\end{array}$ & $\begin{array}{c}0 \\
(0.00)\end{array}$ & $\begin{array}{c}142 \\
(59.16)\end{array}$ & $\begin{array}{c}98 \\
(40.83)\end{array}$ & $\begin{array}{c}240 \\
(100)\end{array}$ \\
\hline
\end{tabular}

Figures in the parentheses indicate the percentages to the total (row wise) 
Table.5 Frequency distribution on the basis of family income of the traditional market customers (Cluster-Pooled)

\begin{tabular}{|c|c|c|c|c|c|c|c|c|c|c|c|}
\hline & \multicolumn{2}{|c|}{$\begin{array}{c}\text { Less than } \\
15000\end{array}$} & \multicolumn{2}{|c|}{$15001-25000$} & \multicolumn{2}{|c|}{$25001-50000$} & \multicolumn{2}{|c|}{$\begin{array}{l}\text { More than } \\
50000\end{array}$} & \multicolumn{2}{|c|}{ Sub Total } & \multirow[t]{2}{*}{ Total } \\
\hline & $\mathbf{M}$ & $\mathbf{F}$ & $\mathbf{M}$ & $\mathbf{F}$ & $\mathbf{M}$ & $\mathbf{F}$ & $\mathbf{M}$ & $\mathbf{F}$ & M & $\mathbf{F}$ & \\
\hline $\begin{array}{l}\text { Cluster- } \\
\text { Sealdah }\end{array}$ & $\begin{array}{c}1 \\
(1.66)\end{array}$ & $\begin{array}{c}1 \\
(1.66)\end{array}$ & $\begin{array}{c}4 \\
(6.66)\end{array}$ & $\begin{array}{c}6 \\
(10)\end{array}$ & $\begin{array}{c}25 \\
(41.66)\end{array}$ & $\begin{array}{c}21 \\
(35)\end{array}$ & $\begin{array}{c}2 \\
(3.33)\end{array}$ & $\begin{array}{c}0 \\
(0.00)\end{array}$ & $\begin{array}{c}32 \\
(53.33)\end{array}$ & $\begin{array}{c}28 \\
(46.66)\end{array}$ & $\begin{array}{c}60 \\
(100)\end{array}$ \\
\hline $\begin{array}{l}\text { Cluster- } \\
\text { Gariahat }\end{array}$ & $\begin{array}{c}0 \\
(0.00)\end{array}$ & $\begin{array}{c}0 \\
(0.00)\end{array}$ & $\begin{array}{c}0 \\
(0.00)\end{array}$ & $\begin{array}{c}2 \\
(5)\end{array}$ & $\begin{array}{c}15 \\
(37.5)\end{array}$ & $\begin{array}{c}9 \\
(22.5)\end{array}$ & $\begin{array}{c}10 \\
(25)\end{array}$ & $\begin{array}{c}2 \\
(5)\end{array}$ & $\begin{array}{c}26 \\
(65)\end{array}$ & $\begin{array}{c}14 \\
(35)\end{array}$ & $\begin{array}{c}40 \\
(100)\end{array}$ \\
\hline $\begin{array}{l}\text { Cluster- } \\
\text { South } \\
\text { Dumdum }\end{array}$ & $\begin{array}{c}0 \\
(0.00)\end{array}$ & $\begin{array}{c}0 \\
(0.00)\end{array}$ & $\begin{array}{c}18 \\
(30)\end{array}$ & $\begin{array}{c}13 \\
(21.66)\end{array}$ & $\begin{array}{c}18 \\
(30)\end{array}$ & $\begin{array}{c}11 \\
(18.33)\end{array}$ & $\begin{array}{c}0 \\
(0.00)\end{array}$ & $\begin{array}{c}0 \\
(0.00)\end{array}$ & $\begin{array}{c}36 \\
(60)\end{array}$ & $\begin{array}{c}24 \\
(40)\end{array}$ & $\begin{array}{c}60 \\
(100)\end{array}$ \\
\hline $\begin{array}{l}\text { Cluster- } \\
\text { Gopalpur }\end{array}$ & $\begin{array}{c}0 \\
(0.00)\end{array}$ & $\begin{array}{c}0 \\
(0.00)\end{array}$ & $\begin{array}{c}0 \\
(0.00)\end{array}$ & $\begin{array}{c}3 \\
(7.5)\end{array}$ & $\begin{array}{c}16 \\
(40)\end{array}$ & $\begin{array}{c}9 \\
(22.5)\end{array}$ & $\begin{array}{c}9 \\
(22.5)\end{array}$ & $\begin{array}{c}2 \\
(5)\end{array}$ & $\begin{array}{c}27 \\
(67.5)\end{array}$ & $\begin{array}{c}13 \\
(32.5)\end{array}$ & $\begin{array}{c}40 \\
(100)\end{array}$ \\
\hline $\begin{array}{l}\text { Cluster- } \\
\text { Newtown }\end{array}$ & $\begin{array}{c}3 \\
(7.5)\end{array}$ & $\begin{array}{c}2 \\
(5)\end{array}$ & $\begin{array}{c}3 \\
(7.5)\end{array}$ & $\begin{array}{c}3 \\
(7.5)\end{array}$ & $\begin{array}{c}15 \\
(37.5)\end{array}$ & $\begin{array}{c}13 \\
(32.5)\end{array}$ & $\begin{array}{c}0 \\
(0.00)\end{array}$ & $\begin{array}{c}1 \\
(2.5)\end{array}$ & $\begin{array}{c}21 \\
(52.5)\end{array}$ & $\begin{array}{c}19 \\
(47.5)\end{array}$ & $\begin{array}{c}40 \\
(100)\end{array}$ \\
\hline $\begin{array}{c}\text { Grand } \\
\text { Total }\end{array}$ & $\begin{array}{c}4 \\
(1.66)\end{array}$ & $\begin{array}{c}3 \\
(1.25)\end{array}$ & $\begin{array}{c}25 \\
(10.41)\end{array}$ & $\begin{array}{c}27 \\
(11.25)\end{array}$ & $\begin{array}{c}89 \\
(37.08)\end{array}$ & $\begin{array}{c}63 \\
(26.25)\end{array}$ & $\begin{array}{c}21 \\
(8.75)\end{array}$ & $\begin{array}{c}3 \\
(1.25)\end{array}$ & $\begin{array}{c}142 \\
(59.16)\end{array}$ & $\begin{array}{c}98 \\
(40.83)\end{array}$ & $\begin{array}{c}240 \\
(100)\end{array}$ \\
\hline
\end{tabular}

Figures in the parentheses indicate the percentages to the total (row wise)

Table.6 Frequency distribution of the traditional market customers on the basis of distance between the residence and traditional market

\begin{tabular}{|c|c|c|c|c|c|c|c|c|c|c|c|c|c|}
\hline & \multicolumn{2}{|c|}{$\begin{array}{c}\text { Less than } \\
0.5 \mathrm{~km}\end{array}$} & \multicolumn{2}{|c|}{$0.5-0.9 \mathrm{~km}$} & \multicolumn{2}{|c|}{$1-1.9 \mathrm{~km}$} & \multicolumn{2}{|c|}{$2-2.9 \mathrm{~km}$} & \multicolumn{2}{|c|}{$\begin{array}{c}\text { More than } \\
3 \mathbf{k m}\end{array}$} & \multicolumn{2}{|c|}{ Sub Total } & \multirow[t]{2}{*}{ Total } \\
\hline & $\mathbf{M}$ & $\mathbf{F}$ & $\mathbf{M}$ & $\mathbf{F}$ & $\mathbf{M}$ & $\mathbf{F}$ & M & $\mathbf{F}$ & $\mathbf{M}$ & $\mathbf{F}$ & M & $\mathbf{F}$ & \\
\hline $\begin{array}{l}\text { Cluster- } \\
\text { Sealdah }\end{array}$ & $\begin{array}{c}0 \\
(0.00)\end{array}$ & $\begin{array}{c}0 \\
(0.00)\end{array}$ & $\begin{array}{c}1 \\
(1.66)\end{array}$ & $\begin{array}{c}1 \\
(1.66)\end{array}$ & $\begin{array}{c}12 \\
(20)\end{array}$ & $\begin{array}{c}12 \\
(20)\end{array}$ & $\begin{array}{c}19 \\
(31.66)\end{array}$ & $\begin{array}{c}15 \\
(25)\end{array}$ & $\begin{array}{c}0 \\
(0.00)\end{array}$ & $\begin{array}{c}0 \\
(0.00)\end{array}$ & $\begin{array}{c}32 \\
(53.33)\end{array}$ & $\begin{array}{c}28 \\
(46.66)\end{array}$ & $\begin{array}{c}60 \\
(100)\end{array}$ \\
\hline $\begin{array}{l}\text { Cluster- } \\
\text { Gariahat }\end{array}$ & $\begin{array}{c}0 \\
(0.00)\end{array}$ & $\begin{array}{c}0 \\
(0.00)\end{array}$ & $\begin{array}{c}6 \\
(15)\end{array}$ & $\begin{array}{c}1 \\
(2.5)\end{array}$ & $\begin{array}{c}11 \\
(27.5)\end{array}$ & $\begin{array}{c}9 \\
(22.5)\end{array}$ & $\begin{array}{c}9 \\
(22.5)\end{array}$ & $\begin{array}{c}4 \\
(10)\end{array}$ & $\begin{array}{c}0 \\
(0.00)\end{array}$ & $\begin{array}{c}0 \\
(0.00)\end{array}$ & $\begin{array}{c}26 \\
(65)\end{array}$ & $\begin{array}{c}14 \\
(35)\end{array}$ & $\begin{array}{c}40 \\
(100)\end{array}$ \\
\hline $\begin{array}{l}\text { Cluster- } \\
\text { South } \\
\text { Dumdum }\end{array}$ & $\begin{array}{c}0 \\
(0.00)\end{array}$ & $\begin{array}{c}0 \\
(0.00)\end{array}$ & $\begin{array}{c}10 \\
(16.66)\end{array}$ & $\begin{array}{c}1 \\
(1.66)\end{array}$ & $\begin{array}{c}9 \\
(15)\end{array}$ & $\begin{array}{c}9 \\
(15)\end{array}$ & $\begin{array}{c}19 \\
(31.66)\end{array}$ & $\begin{array}{c}13 \\
(21.66)\end{array}$ & $\begin{array}{c}0 \\
(0.00)\end{array}$ & $\begin{array}{c}0 \\
(0.00)\end{array}$ & $\begin{array}{c}36 \\
(60)\end{array}$ & $\begin{array}{c}24 \\
(40)\end{array}$ & $\begin{array}{c}60 \\
(100)\end{array}$ \\
\hline $\begin{array}{l}\text { Cluster- } \\
\text { Gopalpur }\end{array}$ & $\begin{array}{c}0 \\
(0.00)\end{array}$ & $\begin{array}{c}0 \\
(0.00)\end{array}$ & $\begin{array}{c}7 \\
(17.5)\end{array}$ & $\begin{array}{c}2 \\
(5)\end{array}$ & $\begin{array}{c}11 \\
(27.5)\end{array}$ & $\begin{array}{c}9 \\
(22.5)\end{array}$ & $\begin{array}{c}9 \\
(22.5)\end{array}$ & $\begin{array}{c}2 \\
(5)\end{array}$ & $\begin{array}{c}0 \\
(0.00)\end{array}$ & $\begin{array}{c}0 \\
(0.00)\end{array}$ & $\begin{array}{c}27 \\
(67.5)\end{array}$ & $\begin{array}{c}13 \\
(32.5)\end{array}$ & $\begin{array}{c}40 \\
(100)\end{array}$ \\
\hline $\begin{array}{l}\text { Cluster- } \\
\text { Newtown }\end{array}$ & $\begin{array}{c}0 \\
(0.00)\end{array}$ & $\begin{array}{c}0 \\
(0.00)\end{array}$ & $\begin{array}{c}1 \\
(2.5)\end{array}$ & $\begin{array}{c}1 \\
(2.5)\end{array}$ & $\begin{array}{c}11 \\
(27.5)\end{array}$ & $\begin{array}{c}9 \\
(22.5)\end{array}$ & $\begin{array}{c}9 \\
(22.5)\end{array}$ & $\begin{array}{c}9 \\
(22.5)\end{array}$ & $\begin{array}{c}0 \\
(0.00)\end{array}$ & $\begin{array}{c}0 \\
(0.00)\end{array}$ & $\begin{array}{c}21 \\
(52.5)\end{array}$ & $\begin{array}{c}19 \\
(47.5)\end{array}$ & $\begin{array}{c}40 \\
(100)\end{array}$ \\
\hline $\begin{array}{l}\text { Grand } \\
\text { Total }\end{array}$ & $\begin{array}{c}0 \\
(0.00)\end{array}$ & $\begin{array}{c}0 \\
(0.00)\end{array}$ & $\begin{array}{c}25 \\
(10.41)\end{array}$ & $\begin{array}{c}6 \\
(2.5)\end{array}$ & $\begin{array}{c}54 \\
(22.5)\end{array}$ & $\begin{array}{c}48 \\
(20)\end{array}$ & $\begin{array}{c}65 \\
(27.08)\end{array}$ & $\begin{array}{c}42 \\
(17.5)\end{array}$ & $\begin{array}{c}0 \\
(0.00)\end{array}$ & $\begin{array}{c}0 \\
(0.00)\end{array}$ & $\begin{array}{c}142 \\
(59.16)\end{array}$ & $\begin{array}{c}98 \\
(40.83)\end{array}$ & $\begin{array}{c}240 \\
(100)\end{array}$ \\
\hline
\end{tabular}

Figures in the parentheses indicate the percentages to the total (row wise) 
Table.7 Frequency distribution traditional market customers on the basis of type of commodities bought by them (Cluster-Pooled)

\begin{tabular}{|c|c|c|c|c|c|c|c|c|c|}
\hline & \multicolumn{2}{|c|}{ Wet } & \multicolumn{2}{|c|}{ Dry } & \multicolumn{2}{|c|}{ Both } & \multicolumn{2}{|c|}{ Sub Total } & \multirow[t]{2}{*}{ Total } \\
\hline & M & $\mathbf{F}$ & M & $\mathbf{F}$ & M & $\mathbf{F}$ & M & $\mathbf{F}$ & \\
\hline $\begin{array}{l}\text { Cluster- } \\
\text { Sealdah }\end{array}$ & $\begin{array}{c}5 \\
(8.33)\end{array}$ & $\begin{array}{c}17 \\
(28.33)\end{array}$ & $\begin{array}{c}12 \\
(20)\end{array}$ & $\begin{array}{c}5 \\
(8.33)\end{array}$ & $\begin{array}{c}10 \\
(16.66)\end{array}$ & $\begin{array}{c}11 \\
(18.33)\end{array}$ & $\begin{array}{c}32 \\
(53.33)\end{array}$ & $\begin{array}{c}28 \\
(46.66)\end{array}$ & $\begin{array}{c}60 \\
(100)\end{array}$ \\
\hline $\begin{array}{l}\text { Cluster- } \\
\text { Gariahat }\end{array}$ & $\begin{array}{c}4 \\
(10)\end{array}$ & $\begin{array}{c}5 \\
(12.5)\end{array}$ & $\begin{array}{c}8 \\
(20)\end{array}$ & $\begin{array}{c}8 \\
(20)\end{array}$ & $\begin{array}{l}10 \\
(25)\end{array}$ & $\begin{array}{c}5 \\
(12.5)\end{array}$ & $\begin{array}{c}26 \\
(65)\end{array}$ & $\begin{array}{c}14 \\
(35)\end{array}$ & $\begin{array}{c}40 \\
(100)\end{array}$ \\
\hline $\begin{array}{l}\text { Cluster-South } \\
\text { Dumdum }\end{array}$ & $\begin{array}{c}16 \\
(26.66)\end{array}$ & $\begin{array}{c}14 \\
(23.33)\end{array}$ & $\begin{array}{c}14 \\
(23.33)\end{array}$ & $\begin{array}{c}6 \\
(10)\end{array}$ & $\begin{array}{c}6 \\
(10)\end{array}$ & $\begin{array}{c}4 \\
(6.66)\end{array}$ & $\begin{array}{c}36 \\
(60)\end{array}$ & $\begin{array}{c}24 \\
(40)\end{array}$ & $\begin{array}{c}60 \\
(100)\end{array}$ \\
\hline $\begin{array}{l}\text { Cluster- } \\
\text { Gopalpur }\end{array}$ & $\begin{array}{c}5 \\
(12.5)\end{array}$ & $\begin{array}{c}5 \\
(12.5)\end{array}$ & $\begin{array}{c}8 \\
(20)\end{array}$ & $\begin{array}{c}8 \\
(20)\end{array}$ & $\begin{array}{l}10 \\
(25)\end{array}$ & $\begin{array}{c}4 \\
(10)\end{array}$ & $\begin{array}{c}27 \\
(67.5)\end{array}$ & $\begin{array}{c}13 \\
(32.5)\end{array}$ & $\begin{array}{c}40 \\
(100)\end{array}$ \\
\hline $\begin{array}{l}\text { Cluster- } \\
\text { Newtown }\end{array}$ & $\begin{array}{c}10 \\
(25)\end{array}$ & $\begin{array}{c}9 \\
(22.5)\end{array}$ & $\begin{array}{c}7 \\
(17.5)\end{array}$ & $\begin{array}{c}7 \\
(17.5)\end{array}$ & $\begin{array}{c}4 \\
(10)\end{array}$ & $\begin{array}{c}3 \\
(7.5)\end{array}$ & $\begin{array}{c}21 \\
(52.5)\end{array}$ & $\begin{array}{c}19 \\
(47.5)\end{array}$ & $\begin{array}{c}40 \\
(100)\end{array}$ \\
\hline Grand Total & $\begin{array}{c}40 \\
(16.66)\end{array}$ & $\begin{array}{c}50 \\
(20.83)\end{array}$ & $\begin{array}{c}49 \\
(20.41)\end{array}$ & $\begin{array}{c}34 \\
(14.16)\end{array}$ & $\begin{array}{c}40 \\
(16.66)\end{array}$ & $\begin{array}{c}27 \\
(11.25)\end{array}$ & $\begin{array}{c}142 \\
(59.16)\end{array}$ & $\begin{array}{c}98 \\
(40.83)\end{array}$ & $\begin{array}{c}240 \\
(100)\end{array}$ \\
\hline
\end{tabular}

Figures in the parentheses indicate the percentages to the total (row wise)

Table.8 Frequency distribution of traditional market customers on the basis of the number of commodities bought by them (Cluster-Pooled)

\begin{tabular}{|c|c|c|c|c|c|c|c|c|c|}
\hline & \multicolumn{2}{|c|}{$\begin{array}{c}\text { Up to } 5 \\
\text { commodities }\end{array}$} & \multicolumn{2}{|c|}{$\begin{array}{c}\text { 5-10 } \\
\text { commodities }\end{array}$} & \multicolumn{2}{|c|}{$\begin{array}{c}\text { More than } 10 \\
\text { commodities }\end{array}$} & \multicolumn{2}{|c|}{ Sub Total } & \multirow[t]{2}{*}{ Total } \\
\hline & $\mathbf{M}$ & $\mathbf{F}$ & $\mathbf{M}$ & $\mathbf{F}$ & M & $\mathbf{F}$ & $\mathbf{M}$ & $\mathbf{F}$ & \\
\hline $\begin{array}{l}\text { Cluster- } \\
\text { Sealdah }\end{array}$ & $\begin{array}{c}3 \\
(5)\end{array}$ & $\begin{array}{c}7 \\
(11.66)\end{array}$ & $\begin{array}{c}16 \\
(26.66)\end{array}$ & $\begin{array}{c}14 \\
(23.33)\end{array}$ & $\begin{array}{c}13 \\
(21.66)\end{array}$ & $\begin{array}{c}7 \\
(11.66)\end{array}$ & $\begin{array}{c}32 \\
(53.33)\end{array}$ & $\begin{array}{c}28 \\
(46.66)\end{array}$ & $\begin{array}{c}60 \\
(100)\end{array}$ \\
\hline $\begin{array}{l}\text { Cluster- } \\
\text { Gariahat }\end{array}$ & $\begin{array}{c}4 \\
(10)\end{array}$ & $\begin{array}{c}6 \\
(15)\end{array}$ & $\begin{array}{c}11 \\
(27.5)\end{array}$ & $\begin{array}{c}8 \\
(20)\end{array}$ & $\begin{array}{c}11 \\
(27.5)\end{array}$ & $\begin{array}{c}0 \\
(0.00)\end{array}$ & $\begin{array}{c}26 \\
(65)\end{array}$ & $\begin{array}{c}14 \\
(35)\end{array}$ & $\begin{array}{c}40 \\
(100)\end{array}$ \\
\hline $\begin{array}{l}\text { Cluster-South } \\
\text { Dumdum }\end{array}$ & $\begin{array}{c}8 \\
(13.33)\end{array}$ & $\begin{array}{c}8 \\
(13.33)\end{array}$ & $\begin{array}{c}15 \\
(25)\end{array}$ & $\begin{array}{c}10 \\
(16.66)\end{array}$ & $\begin{array}{c}13 \\
(21.66)\end{array}$ & $\begin{array}{c}6 \\
(10)\end{array}$ & $\begin{array}{c}36 \\
(60)\end{array}$ & $\begin{array}{c}24 \\
(40)\end{array}$ & $\begin{array}{c}60 \\
(100)\end{array}$ \\
\hline $\begin{array}{l}\text { Cluster- } \\
\text { Gopalpur }\end{array}$ & $\begin{array}{c}5 \\
(12.5)\end{array}$ & $\begin{array}{c}5 \\
(12.5)\end{array}$ & $\begin{array}{c}11 \\
(27.5)\end{array}$ & $\begin{array}{c}9 \\
(22.5)\end{array}$ & $\begin{array}{c}10 \\
(25)\end{array}$ & $\begin{array}{c}0 \\
(0.00)\end{array}$ & $\begin{array}{c}27 \\
(67.5)\end{array}$ & $\begin{array}{c}13 \\
(32.5)\end{array}$ & $\begin{array}{c}40 \\
(100)\end{array}$ \\
\hline $\begin{array}{l}\text { Cluster- } \\
\text { Newtown }\end{array}$ & $\begin{array}{c}3 \\
(7.5)\end{array}$ & $\begin{array}{c}6 \\
(15)\end{array}$ & $\begin{array}{c}9 \\
(22.5)\end{array}$ & $\begin{array}{c}13 \\
(32.5)\end{array}$ & $\begin{array}{c}6 \\
(15)\end{array}$ & $\begin{array}{c}3 \\
(7.5)\end{array}$ & $\begin{array}{c}21 \\
(52.5)\end{array}$ & $\begin{array}{c}19 \\
(47.5)\end{array}$ & $\begin{array}{c}40 \\
(100)\end{array}$ \\
\hline Grand Total & $\begin{array}{c}23 \\
(9.58)\end{array}$ & $\begin{array}{c}32 \\
(13.33)\end{array}$ & $\begin{array}{c}62 \\
(25.83)\end{array}$ & $\begin{array}{c}54 \\
(22.50)\end{array}$ & $\begin{array}{c}53 \\
(22.08)\end{array}$ & $\begin{array}{c}16 \\
(6.66)\end{array}$ & $\begin{array}{c}142 \\
(59.16)\end{array}$ & $\begin{array}{c}98 \\
(40.83)\end{array}$ & $\begin{array}{c}240 \\
(100)\end{array}$ \\
\hline
\end{tabular}

Figures in the parentheses indicate the percentages to the total (row wise) 
Table.9 Frequency distribution of the traditional market customers on the basis of expenditure made by them (Cluster-Pooled)

\begin{tabular}{|c|c|c|c|c|c|c|c|c|c|}
\hline & \multicolumn{2}{|c|}{ Up to Rs 500} & \multicolumn{2}{|c|}{$500-1000$} & \multicolumn{2}{|c|}{ More than 1000} & \multicolumn{2}{|c|}{ Sub Total } & \multirow[t]{2}{*}{ Total } \\
\hline & $\mathbf{M}$ & $\mathbf{F}$ & $\mathbf{M}$ & $\mathbf{F}$ & $\mathbf{M}$ & $\mathbf{F}$ & $\mathbf{M}$ & $\mathbf{F}$ & \\
\hline $\begin{array}{l}\text { Cluster- } \\
\text { Sealdah }\end{array}$ & $\begin{array}{c}4 \\
(6.66)\end{array}$ & $\begin{array}{c}8 \\
(13.33)\end{array}$ & $\begin{array}{c}20 \\
(33.33)\end{array}$ & $\begin{array}{c}9 \\
(15)\end{array}$ & $\begin{array}{c}9 \\
(15)\end{array}$ & $\begin{array}{c}10 \\
(16.66)\end{array}$ & $\begin{array}{c}32 \\
(53.33)\end{array}$ & $\begin{array}{c}28 \\
(46.66)\end{array}$ & $\begin{array}{c}60 \\
(100)\end{array}$ \\
\hline $\begin{array}{l}\text { Cluster- } \\
\text { Gariahat }\end{array}$ & $\begin{array}{c}4 \\
(10)\end{array}$ & $\begin{array}{c}6 \\
(15)\end{array}$ & $\begin{array}{c}16 \\
(40)\end{array}$ & $\begin{array}{c}8 \\
(20)\end{array}$ & $\begin{array}{c}1 \\
(2.5)\end{array}$ & $\begin{array}{c}5 \\
(12.5)\end{array}$ & $\begin{array}{c}26 \\
(65)\end{array}$ & $\begin{array}{c}14 \\
(35)\end{array}$ & $\begin{array}{c}40 \\
(100)\end{array}$ \\
\hline $\begin{array}{l}\text { Cluster- } \\
\text { South } \\
\text { Dumdum }\end{array}$ & $\begin{array}{c}6 \\
(10)\end{array}$ & $\begin{array}{c}9 \\
(15)\end{array}$ & $\begin{array}{c}20 \\
(33.33)\end{array}$ & $\begin{array}{c}7 \\
(11.66)\end{array}$ & $\begin{array}{c}10 \\
(16.66)\end{array}$ & $\begin{array}{c}8 \\
(13.33)\end{array}$ & $\begin{array}{c}36 \\
(60)\end{array}$ & $\begin{array}{c}24 \\
(40)\end{array}$ & $\begin{array}{c}60 \\
(100)\end{array}$ \\
\hline $\begin{array}{l}\text { Cluster- } \\
\text { Gopalpur }\end{array}$ & $\begin{array}{c}8 \\
(20)\end{array}$ & $\begin{array}{c}6 \\
(15)\end{array}$ & $\begin{array}{c}15 \\
(37.5)\end{array}$ & $\begin{array}{c}2 \\
(5)\end{array}$ & $\begin{array}{c}4 \\
(10)\end{array}$ & $\begin{array}{c}5 \\
(12.5)\end{array}$ & $\begin{array}{c}27 \\
(67.5)\end{array}$ & $\begin{array}{c}13 \\
(32.5)\end{array}$ & $\begin{array}{c}40 \\
(100)\end{array}$ \\
\hline $\begin{array}{l}\text { Cluster- } \\
\text { Newtown }\end{array}$ & $\begin{array}{c}3 \\
(7.5)\end{array}$ & $\begin{array}{c}6 \\
(15)\end{array}$ & $\begin{array}{c}14 \\
(35)\end{array}$ & $\begin{array}{c}8 \\
(20)\end{array}$ & $\begin{array}{c}2 \\
(5)\end{array}$ & $\begin{array}{c}7 \\
(17.5)\end{array}$ & $\begin{array}{c}21 \\
(52.5)\end{array}$ & $\begin{array}{c}19 \\
(47.5)\end{array}$ & $\begin{array}{c}40 \\
(100)\end{array}$ \\
\hline Grand Total & $\begin{array}{c}25 \\
(10.41)\end{array}$ & $\begin{array}{c}35 \\
(14.58)\end{array}$ & $\begin{array}{c}85 \\
(35.41)\end{array}$ & $\begin{array}{c}34 \\
(14.16)\end{array}$ & $\begin{array}{c}26 \\
(10.83)\end{array}$ & $\begin{array}{c}35 \\
(14.58)\end{array}$ & $\begin{array}{c}142 \\
(59.16)\end{array}$ & $\begin{array}{c}98 \\
(40.83)\end{array}$ & $\begin{array}{c}240 \\
(100)\end{array}$ \\
\hline
\end{tabular}

Figures in the parentheses indicate the percentages to the total (row wise)

The Table 8 demonstrates the frequency distribution of the traditional market customer of all the traditional markets on the basis of number of commodities bought by them across all the clusters in Kolkata. It is found from the table that the maximum percentage of customers purchases 5 to 10 number of commodities followed by the percentage of consumers who purchases more than 10 commodities from the selected traditional markets.

The Table 9 demonstrates the frequency distribution of the traditional market customers on the basis of type of expenditure made by them in all the traditional markets across all the clusters in Kolkata. It is evident from the table that, the maximum percentage of customers' purchases commodities within price range of rupees 500 to 1000 followed price range of more than 1000 rupees.
The Table 10 reveals the frequency distribution of the traditional market customers on the basis of type of accompany the customers prefers while visiting the selected market across all the clusters of Kolkata. From the table it is understood from the table that in the traditional markets, the customers there prefers to come alone for purchasing of commodities.

The Table 11 reveals the frequency distribution of the customers of organized outlets on the basis of time interval of purchasing wet commodities from organized outlets across all the clusters in Kolkata. It is observed from the table that, the maximum percentage of customers' purchases wet commodities (vegetables and fruits) from organized retail outlets once in a month followed by customers who purchases more than once in a month from organized outlets. 
Table.10 Frequency distribution of the traditional market customers on the basis of type of accompany (Cluster-Pooled)

\begin{tabular}{|c|c|c|c|c|c|c|c|c|c|c|c|c|c|}
\hline & \multicolumn{2}{|c|}{ Alone } & \multicolumn{2}{|c|}{ Couple } & \multicolumn{2}{|c|}{ Family } & \multicolumn{2}{|c|}{ Friends } & \multicolumn{2}{|c|}{ Neighbors } & \multicolumn{2}{|c|}{ Sub Total } & \multirow[t]{2}{*}{ Total } \\
\hline & $\mathbf{M}$ & $\mathbf{F}$ & M & F & M & F & $\mathbf{M}$ & $\mathbf{F}$ & M & F & $\mathbf{M}$ & F & \\
\hline $\begin{array}{l}\text { Cluster- } \\
\text { Sealdah }\end{array}$ & $\begin{array}{c}13 \\
(21.66)\end{array}$ & $\begin{array}{c}15 \\
(25)\end{array}$ & $\begin{array}{c}10 \\
(16.66)\end{array}$ & $\begin{array}{c}15 \\
(25)\end{array}$ & $\begin{array}{c}4 \\
(6.66)\end{array}$ & $\begin{array}{c}3 \\
(5)\end{array}$ & $\begin{array}{c}0 \\
(0.00)\end{array}$ & $\begin{array}{c}0 \\
(0.00)\end{array}$ & $\begin{array}{c}0 \\
(0.00)\end{array}$ & $\begin{array}{c}0 \\
(0.00)\end{array}$ & $\begin{array}{c}32 \\
(53.33)\end{array}$ & $\begin{array}{c}28 \\
(46.66)\end{array}$ & $\begin{array}{c}60 \\
(100)\end{array}$ \\
\hline $\begin{array}{l}\text { Cluster- } \\
\text { Gariahat }\end{array}$ & $\begin{array}{c}13 \\
(32.5)\end{array}$ & $\begin{array}{c}7 \\
(17.5)\end{array}$ & $\begin{array}{c}8 \\
(20)\end{array}$ & $\begin{array}{c}6 \\
(15)\end{array}$ & $\begin{array}{c}5 \\
(12.5)\end{array}$ & $\begin{array}{c}1 \\
(2.5)\end{array}$ & $\begin{array}{c}0 \\
(0.00)\end{array}$ & $\begin{array}{c}0 \\
(0.00)\end{array}$ & $\begin{array}{c}0 \\
(0.00)\end{array}$ & $\begin{array}{c}0 \\
(0.00)\end{array}$ & $\begin{array}{c}26 \\
(65)\end{array}$ & $\begin{array}{c}14 \\
(35)\end{array}$ & $\begin{array}{c}40 \\
(100)\end{array}$ \\
\hline $\begin{array}{l}\text { Cluster-South } \\
\text { Dumdum }\end{array}$ & $\begin{array}{c}20 \\
(33.33)\end{array}$ & $\begin{array}{c}16 \\
(26.66)\end{array}$ & $\begin{array}{c}9 \\
(15)\end{array}$ & $\begin{array}{c}8 \\
(13.33)\end{array}$ & $\begin{array}{c}7 \\
(11.66)\end{array}$ & $\begin{array}{c}0 \\
(0.00)\end{array}$ & $\begin{array}{c}0 \\
(0.00)\end{array}$ & $\begin{array}{c}0 \\
(0.00)\end{array}$ & $\begin{array}{c}0 \\
(0.00)\end{array}$ & $\begin{array}{c}0 \\
(0.00)\end{array}$ & $\begin{array}{c}36 \\
(60)\end{array}$ & $\begin{array}{c}24 \\
(40)\end{array}$ & $\begin{array}{c}60 \\
(100)\end{array}$ \\
\hline $\begin{array}{c}\text { Cluster- } \\
\text { Gopalpur }\end{array}$ & $\begin{array}{c}14 \\
(35)\end{array}$ & $\begin{array}{c}8 \\
(20)\end{array}$ & $\begin{array}{c}8 \\
(20)\end{array}$ & $\begin{array}{c}5 \\
(12.5)\end{array}$ & $\begin{array}{c}5 \\
(12.5)\end{array}$ & $\begin{array}{c}0 \\
(0.00)\end{array}$ & $\begin{array}{c}0 \\
(0.00)\end{array}$ & $\begin{array}{c}0 \\
(0.00)\end{array}$ & $\begin{array}{c}0 \\
(0.00)\end{array}$ & $\begin{array}{c}0 \\
(0.00)\end{array}$ & $\begin{array}{c}27 \\
(67.5)\end{array}$ & $\begin{array}{c}13 \\
(32.5)\end{array}$ & $\begin{array}{c}40 \\
(100)\end{array}$ \\
\hline $\begin{array}{l}\text { Cluster- } \\
\text { Newtown }\end{array}$ & $\begin{array}{c}5 \\
(12.5)\end{array}$ & $\begin{array}{c}7 \\
(17.5)\end{array}$ & $\begin{array}{c}8 \\
(20)\end{array}$ & $\begin{array}{c}6 \\
(15)\end{array}$ & $\begin{array}{c}8 \\
(20)\end{array}$ & $\begin{array}{c}6 \\
(15)\end{array}$ & $\begin{array}{c}0 \\
(0.00)\end{array}$ & $\begin{array}{c}0 \\
(0.00)\end{array}$ & $\begin{array}{c}0 \\
(0.00)\end{array}$ & $\begin{array}{c}0 \\
(0.00)\end{array}$ & $\begin{array}{c}21 \\
(52.5)\end{array}$ & $\begin{array}{c}19 \\
(47.5)\end{array}$ & $\begin{array}{c}40 \\
(100)\end{array}$ \\
\hline Grand Total & $\begin{array}{c}65 \\
(27.08)\end{array}$ & $\begin{array}{c}53 \\
(22.08)\end{array}$ & $\begin{array}{c}43 \\
(17.91)\end{array}$ & $\begin{array}{c}40 \\
(16.66)\end{array}$ & $\begin{array}{c}29 \\
(12.08)\end{array}$ & $\begin{array}{c}10 \\
(4.16)\end{array}$ & $\begin{array}{c}0 \\
(0.00)\end{array}$ & $\begin{array}{c}0 \\
(0.00)\end{array}$ & $\begin{array}{c}0 \\
(0.00)\end{array}$ & $\begin{array}{c}0 \\
(0.00)\end{array}$ & $\begin{array}{c}142 \\
(59.16)\end{array}$ & $\begin{array}{c}98 \\
(40.83)\end{array}$ & $\begin{array}{c}240 \\
(100)\end{array}$ \\
\hline
\end{tabular}

Figures in the parentheses indicate the percentages to the total (row wise)

Table.11 Frequency distribution of the traditional market customers on the basis of purchasing wet commodities from organized outlets

\begin{tabular}{|c|c|c|c|c|c|c|c|c|c|c|c|c|c|}
\hline & \multicolumn{2}{|c|}{ Daily } & \multicolumn{2}{|c|}{ Weekend } & \multicolumn{2}{|c|}{ Once in a Month } & \multicolumn{2}{|c|}{$\begin{array}{c}\text { More than Once in a } \\
\text { Month }\end{array}$} & \multicolumn{2}{|c|}{ Never } & \multicolumn{2}{|c|}{ Sub Total } & \multirow[t]{2}{*}{ Total } \\
\hline & M & $\mathbf{F}$ & M & $\mathbf{F}$ & M & F & M & F & $\mathbf{M}$ & $\mathbf{F}$ & $\mathbf{M}$ & F & \\
\hline $\begin{array}{l}\text { Cluster- } \\
\text { Sealdah }\end{array}$ & $\begin{array}{c}0 \\
(0.00)\end{array}$ & $\begin{array}{c}0 \\
(0.00)\end{array}$ & $\begin{array}{c}0 \\
(0.00)\end{array}$ & $\begin{array}{c}0 \\
(0.00)\end{array}$ & $\begin{array}{c}5 \\
(8.33) \\
\end{array}$ & $\begin{array}{c}8 \\
(13.33)\end{array}$ & $\begin{array}{c}0 \\
(0.00)\end{array}$ & $\begin{array}{c}0 \\
(0.00)\end{array}$ & $\begin{array}{c}27 \\
(45)\end{array}$ & $\begin{array}{c}20 \\
(33.33)\end{array}$ & $\begin{array}{c}32 \\
(53.33)\end{array}$ & $\begin{array}{c}28 \\
(46.66)\end{array}$ & $\begin{array}{c}60 \\
(100)\end{array}$ \\
\hline $\begin{array}{l}\text { Cluster- } \\
\text { Gariahat }\end{array}$ & $\begin{array}{c}0 \\
(0.00)\end{array}$ & $\begin{array}{c}0 \\
(0.00)\end{array}$ & $\begin{array}{c}0 \\
(0.00)\end{array}$ & $\begin{array}{c}0 \\
(0.00)\end{array}$ & $\begin{array}{c}17 \\
(42.5)\end{array}$ & $\begin{array}{c}8 \\
(20)\end{array}$ & $\begin{array}{c}0 \\
(0.00)\end{array}$ & $\begin{array}{c}1 \\
(2.5)\end{array}$ & $\begin{array}{c}9 \\
(22.5)\end{array}$ & $\begin{array}{c}5 \\
(12.5)\end{array}$ & $\begin{array}{c}26 \\
(65)\end{array}$ & $\begin{array}{c}14 \\
(35)\end{array}$ & $\begin{array}{c}40 \\
(100)\end{array}$ \\
\hline $\begin{array}{l}\text { Cluster-South } \\
\text { Dumdum }\end{array}$ & $\begin{array}{c}0 \\
(0.00)\end{array}$ & $\begin{array}{c}0 \\
(0.00)\end{array}$ & $\begin{array}{c}0 \\
(0.00)\end{array}$ & $\begin{array}{c}0 \\
(0.00)\end{array}$ & $\begin{array}{l}15 \\
(25)\end{array}$ & $\begin{array}{c}11 \\
(18.33)\end{array}$ & $\begin{array}{c}13 \\
(21.66)\end{array}$ & $\begin{array}{c}6 \\
(10)\end{array}$ & $\begin{array}{c}8 \\
(13.33)\end{array}$ & $\begin{array}{c}7 \\
(11.66)\end{array}$ & $\begin{array}{c}36 \\
(60)\end{array}$ & $\begin{array}{c}24 \\
(40)\end{array}$ & $\begin{array}{c}60 \\
(100)\end{array}$ \\
\hline $\begin{array}{l}\text { Cluster- } \\
\text { Gopalpur }\end{array}$ & $\begin{array}{c}0 \\
(0.00)\end{array}$ & $\begin{array}{c}0 \\
(0.00)\end{array}$ & $\begin{array}{c}0 \\
(0.00)\end{array}$ & $\begin{array}{c}0 \\
(0.00)\end{array}$ & $\begin{array}{c}18 \\
(45)\end{array}$ & $\begin{array}{c}7 \\
(17.5)\end{array}$ & $\begin{array}{c}0 \\
(0.00)\end{array}$ & $\begin{array}{c}1 \\
(2.5)\end{array}$ & $\begin{array}{c}9 \\
(22.5)\end{array}$ & $\begin{array}{c}5 \\
(12.5)\end{array}$ & $\begin{array}{c}27 \\
(67.5)\end{array}$ & $\begin{array}{c}13 \\
(32.5)\end{array}$ & $\begin{array}{c}40 \\
(100)\end{array}$ \\
\hline $\begin{array}{l}\text { Cluster- } \\
\text { Newtown }\end{array}$ & $\begin{array}{c}0 \\
(0.00)\end{array}$ & $\begin{array}{c}0 \\
(0.00)\end{array}$ & $\begin{array}{c}0 \\
(0.00)\end{array}$ & $\begin{array}{c}0 \\
(0.00)\end{array}$ & $\begin{array}{c}1 \\
(2.5)\end{array}$ & $\begin{array}{c}3 \\
(7.5)\end{array}$ & $\begin{array}{c}0 \\
(0.00)\end{array}$ & $\begin{array}{c}1 \\
(2.5)\end{array}$ & $\begin{array}{c}20 \\
(50)\end{array}$ & $\begin{array}{c}15 \\
(37.5)\end{array}$ & $\begin{array}{c}21 \\
(52.5)\end{array}$ & $\begin{array}{c}19 \\
(47.5)\end{array}$ & $\begin{array}{c}40 \\
(100)\end{array}$ \\
\hline Grand Total & $\begin{array}{c}0 \\
(0.00)\end{array}$ & $\begin{array}{c}0 \\
(0.00)\end{array}$ & $\begin{array}{c}0 \\
(0.00)\end{array}$ & $\begin{array}{c}0 \\
(0.00)\end{array}$ & $\begin{array}{c}56 \\
(23.33)\end{array}$ & $\begin{array}{c}37 \\
(15.41)\end{array}$ & $\begin{array}{c}13 \\
(5.41)\end{array}$ & $\begin{array}{c}9 \\
(3.75)\end{array}$ & $\begin{array}{c}73 \\
(30.41)\end{array}$ & $\begin{array}{c}52 \\
(21.66)\end{array}$ & $\begin{array}{c}142 \\
(59.16)\end{array}$ & $\begin{array}{c}98 \\
(40.83)\end{array}$ & $\begin{array}{c}240 \\
(100)\end{array}$ \\
\hline
\end{tabular}

Figures in the parentheses indicate the percentages to the total (row wise) 
Table.12 Frequency distribution of the traditional market customers on the basis of purchasing dry commodities from organized outlets

\begin{tabular}{|c|c|c|c|c|c|c|c|c|c|c|c|c|c|}
\hline & \multicolumn{2}{|c|}{ Daily } & \multicolumn{2}{|c|}{ Weekend } & \multicolumn{2}{|c|}{ Once in a Month } & \multicolumn{2}{|c|}{$\begin{array}{l}\text { More than Once } \\
\text { in a Month }\end{array}$} & \multicolumn{2}{|c|}{ Never } & \multicolumn{2}{|c|}{ Sub Total } & \multirow[t]{2}{*}{ Total } \\
\hline & $\mathbf{M}$ & $\mathbf{F}$ & M & $\mathbf{F}$ & M & $\mathbf{F}$ & M & $\mathbf{F}$ & M & $\mathbf{F}$ & $\mathbf{M}$ & $\mathbf{F}$ & \\
\hline $\begin{array}{l}\text { Cluster- } \\
\text { Sealdah }\end{array}$ & $\begin{array}{c}0 \\
(0.00)\end{array}$ & $\begin{array}{c}0 \\
(0.00)\end{array}$ & $\begin{array}{c}0 \\
(0.00)\end{array}$ & $\begin{array}{c}0 \\
(0.00)\end{array}$ & $\begin{array}{c}6 \\
(10)\end{array}$ & $\begin{array}{c}10 \\
(16.66)\end{array}$ & $\begin{array}{c}10 \\
(16.66)\end{array}$ & $\begin{array}{c}4 \\
(6.66)\end{array}$ & $\begin{array}{l}15 \\
(25)\end{array}$ & $\begin{array}{c}15 \\
(25)\end{array}$ & $\begin{array}{c}32 \\
(53.33)\end{array}$ & $\begin{array}{c}28 \\
(46.66)\end{array}$ & $\begin{array}{c}60 \\
(100)\end{array}$ \\
\hline $\begin{array}{l}\text { Cluster- } \\
\text { Gariahat }\end{array}$ & $\begin{array}{c}0 \\
(0.00)\end{array}$ & $\begin{array}{c}0 \\
(0.00)\end{array}$ & $\begin{array}{c}0 \\
(0.00)\end{array}$ & $\begin{array}{c}0 \\
(0.00)\end{array}$ & $\begin{array}{c}18 \\
(45)\end{array}$ & $\begin{array}{c}12 \\
(30)\end{array}$ & $\begin{array}{c}0 \\
(0.00)\end{array}$ & $\begin{array}{c}1 \\
(2.5)\end{array}$ & $\begin{array}{c}7 \\
(17.5)\end{array}$ & $\begin{array}{c}2 \\
(5)\end{array}$ & $\begin{array}{c}26 \\
(65)\end{array}$ & $\begin{array}{c}14 \\
(35)\end{array}$ & $\begin{array}{c}40 \\
(100)\end{array}$ \\
\hline $\begin{array}{l}\text { Cluster- } \\
\text { South } \\
\text { Dumdum }\end{array}$ & $\begin{array}{c}0 \\
(0.00)\end{array}$ & $\begin{array}{c}0 \\
(0.00)\end{array}$ & $\begin{array}{c}0 \\
(0.00)\end{array}$ & $\begin{array}{c}0 \\
(0.00)\end{array}$ & $\begin{array}{c}16 \\
(26.66)\end{array}$ & $\begin{array}{c}10 \\
(16.66)\end{array}$ & $\begin{array}{c}6 \\
(10)\end{array}$ & $\begin{array}{c}4 \\
(6.66)\end{array}$ & $\begin{array}{c}14 \\
(23.33)\end{array}$ & $\begin{array}{c}10 \\
(16.66)\end{array}$ & $\begin{array}{c}36 \\
(60)\end{array}$ & $\begin{array}{c}24 \\
(40)\end{array}$ & $\begin{array}{c}60 \\
(100)\end{array}$ \\
\hline $\begin{array}{l}\text { Cluster- } \\
\text { Gopalpur }\end{array}$ & $\begin{array}{c}0 \\
(0.00)\end{array}$ & $\begin{array}{c}0 \\
(0.00)\end{array}$ & $\begin{array}{c}0 \\
(0.00)\end{array}$ & $\begin{array}{c}0 \\
(0.00)\end{array}$ & $\begin{array}{c}10 \\
(25)\end{array}$ & $\begin{array}{c}3 \\
(7.5)\end{array}$ & $\begin{array}{c}0 \\
(0.00)\end{array}$ & $\begin{array}{c}1 \\
(2.5)\end{array}$ & $\begin{array}{c}15 \\
(25)\end{array}$ & $\begin{array}{c}11 \\
(27.5)\end{array}$ & $\begin{array}{c}27 \\
(67.5)\end{array}$ & $\begin{array}{c}13 \\
(32.5)\end{array}$ & $\begin{array}{c}40 \\
(100)\end{array}$ \\
\hline $\begin{array}{l}\text { Cluster- } \\
\text { Newtown }\end{array}$ & $\begin{array}{c}0 \\
(0.00)\end{array}$ & $\begin{array}{c}0 \\
(0.00)\end{array}$ & $\begin{array}{c}0 \\
(0.00)\end{array}$ & $\begin{array}{c}0 \\
(0.00)\end{array}$ & $\begin{array}{c}7 \\
(17.5)\end{array}$ & $\begin{array}{c}2 \\
(5)\end{array}$ & $\begin{array}{c}0 \\
(0.00)\end{array}$ & $\begin{array}{c}1 \\
(2.5)\end{array}$ & $\begin{array}{c}18 \\
(45)\end{array}$ & $\begin{array}{c}12 \\
(30)\end{array}$ & $\begin{array}{c}21 \\
(52.5)\end{array}$ & $\begin{array}{c}19 \\
(47.5)\end{array}$ & $\begin{array}{c}40 \\
(100)\end{array}$ \\
\hline $\begin{array}{c}\text { Grand } \\
\text { Total }\end{array}$ & $\begin{array}{c}0 \\
(0.00)\end{array}$ & $\begin{array}{c}0 \\
(0.00)\end{array}$ & $\begin{array}{c}0 \\
(0.00)\end{array}$ & $\begin{array}{c}0 \\
(0.00)\end{array}$ & $\begin{array}{c}57 \\
(23.75)\end{array}$ & $\begin{array}{c}37 \\
(15.41)\end{array}$ & $\begin{array}{c}16 \\
(6.66)\end{array}$ & $\begin{array}{c}11 \\
(4.58)\end{array}$ & $\begin{array}{c}69 \\
(28.73)\end{array}$ & $\begin{array}{c}50 \\
(20.83)\end{array}$ & $\begin{array}{c}142 \\
(59.16)\end{array}$ & $\begin{array}{c}98 \\
(40.83)\end{array}$ & $\begin{array}{c}240 \\
(100)\end{array}$ \\
\hline
\end{tabular}

Figures in the parentheses indicate the percentages to the total (row wise)

Table 12 reveals the frequency distribution of the traditional market customers of markets on the basis of time interval of purchasing dry commodities from organized outlets across all the clusters. It can be observed from the table that the maximum percentage of customers prefers purchasing dry commodities (grocery items) once in a month followed by frequency of more than once in a month. The above research study may be concluded that there are total 5 clusters (Sealdah, Gariahat, South Dumdum, Gopalpur, Newtown) taken into consideration in Kolkata for the study.

The age-wise and sex-wise frequency distribution of the traditional market customers in all the traditional markets across all the clusters out of all the age groups, the maximum percentage of the male and female customers belong to 21 to 40 years age group.

The frequency distribution of traditional market customers on the basis of family size in all the clusters shows that all the family sizes are found but the maximum percentage of traditional market customers have family size of 4-6 members.
From the frequency distribution of traditional market customers on the basis on the basis of educational qualification in all the traditional markets across all the clusters, it displays that maximum percentage of customers in traditional market are graduates'.

The frequency distribution of traditional market consumers on the basis of occupation in all the traditional markets across all the clusters shows that the maximum percentages of customers are private sectors service holders.

From the frequency distribution of traditional market consumers on the basis of family income in all the traditional markets across all the clusters it have been observed that the maximum percentage of family income belongs to family income group of Rs 25001 to 50000.

The frequency distribution of the traditional market customers on the basis type of commodities bought by them in all the traditional markets across all the clusters in Kolkata shows that the maximum percentage of consumers purchases wet commodities (vegetables and fruits) from the traditional markets. 
From the frequency distribution of the traditional market customers of all the traditional markets on the basis of number of commodities bought by them across all the clusters in Kolkata, it is found that the maximum percentage of customers purchases 5 to 10 number of commodities from the selected traditional markets.

The frequency distribution of the traditional market customers on the basis of type of expenditure made by them in all the traditional markets across all the clusters in Kolkata shows that the maximum percentage of customers' purchases commodities within price range of rupees 500 to 1000 .

The frequency distribution of the traditional market customers on the basis of type of accompany the customers prefers while visiting the selected market across all the clusters of Kolkata, it is understood that in the traditional markets, the customers there prefers to come alone for purchasing of commodities.

The frequency distribution of the customers of organized outlets on the basis of time interval of purchasing wet commodities from organized outlets across all the clusters in Kolkata shows that the maximum percentage of customers' purchases wet commodities (vegetables and fruits) from organized retail outlets once in a month.

From the frequency distribution of the traditional market customers of markets on the basis of time interval of purchasing dry commodities from organized outlets across all the clusters shows that the maximum percentage of customers prefers purchasing dry commodities (grocery items) once in a month from traditional outlets.

\section{References}

Anand, V. and Gopalakrishnan, P. G. (2011) "A Mantra for the Peaceful CExistence of Malls and Kiranas", SDCM Journal of Management, Issue 3, pp.78-83.

Aravind, S. (2008) "Consumers"e Perception Regarding Organized Grocery Retailing" SCOUR- Journal of Institute of Productivity \& Management, Vol. 02, Number 01, pp. 35-46.

Johnson, K.K.P., Yoo, J.J., Rhee, J., Lennon, S., Jasper, Damhorst (2006) "MultiChannel Shopping: Channel Use Among Rural Consumers" International Journal of Retail \& Distribution Management, Vol. 34, No. 6, pp. 453-466.

Rathod, R.M. and Patel, R. P. (2008), "An Empirical Study on Customers Choice Criteria to select Exclusive and MultiBrand Retail Outlet", Rai Management Journal, Vol.5 (1), pp.34-42.

Thomas and Pathak (2012), "A study of Consumer Behaviour Approach towards Shopping Mall Attractiveness with special reference to the city of Ahmedabad", Research Journal of Social Science and Management, Volume: 02, Number: 05,pp-147-152.

\section{How to cite this article:}

Sinha, S. and Md. H. Ali. 2020. Some Socio-Economic Aspects of Consumers in Unorganized Markets of Kolkata City. Int.J.Curr.Microbiol.App.Sci. 9(04): 1683-1693. doi: https://doi.org/10.20546/ijcmas.2020.904.197 